\title{
Retracing behavior and broadband generation based on quasi-phase-matched optical parametric processes
}

\author{
Chih-Wei Hsu, Chieh-Ting Chen, and Chih-Chung Yang \\ Department of Electrical Engineering and Graduate Institute of Electro-Optical Engineering, National Taiwan \\ University, 1, Roosevelt Road, Section 4, Taipei, Taiwan
}

Received July 23, 2001

\begin{abstract}
Broadband operation based on retracing behavior $(\mathrm{RB})$ of the phase-matching curves in various quasi-phasematched materials with collinear, non-collinear, and quasi-collinear phase-matching schemes is theoretically studied. Broadband operation is feasible only at the degenerate point in a collinear configuration with a specific pump wavelength, $\lambda_{\mathrm{BC}}$. Such a constraint sets a significant limitation on application of that scheme. With a noncollinear or a quasi-collinear phase-matching configuration the conditions and the resultant output signal ranges of broadband operation are much more flexible, particularly those that are useful for fiber communications and optical imaging of biological tissues. In a signal-resonated (idler-resonated) optical parametric oscillator (OPO) with a noncollinear or a quasi-collinear configuration, RB and hence broadband operation can be observed only for pump wavelengths shorter (longer) than $\lambda_{\mathrm{BC}}$. Also, broadband operation for an idler-resonated OPO with a pump wavelength longer than $\lambda_{\mathrm{BC}}$ can be obtained only at the degenerate point. Nevertheless, broadband phenomena for a signal-resonated OPO with a pump wavelength shorter than $\lambda_{\mathrm{BC}}$ can be observed either at or away from the degenerate points. (C) 2002 Optical Society of America
\end{abstract}

OCIS codes: $190.4970,190.4410$.

\section{INTRODUCTION}

Broadband optical sources are important for applications in many areas, including wavelength-division multiplexing signal generation, amplification, and wavelength conversion in fiber communications, optical coherence tomography for medical diagnosis, and spectroscopic research of wide excitation tunability. For wavelength-division multiplexing fiber communications, besides conventional erbium-doped fiber amplification and stimulated Raman scattering in fiber, $\chi^{(2)}$ nonlinear optics has been considered for broadband signal amplification based on engineered quasi-phase matching (QPM) in $\mathrm{LiNbO}_{3} \cdot{ }^{1}$ Also, if broadband operation is available near the degenerate point in a $\chi^{(2)}$ process, wavelength conversion of a wide range of channels can be implemented based on difference-frequency generation. ${ }^{2,3}$ In comparison with erbium-doped fiber amplifiers and semiconductor optical amplifiers, a $\chi^{(2)}$ nonlinear optics process for fiber communication has the advantages of being a high-frequency process and suffering less amplified-spontaneousemission noise and less cross talk. In the application of optical coherence tomography, which is an important technique for medical diagnosis, a broader source bandwidth results in higher spatial resolution in the scanning depth dimension. ${ }^{4}$ Therefore broadband sources are crucially important for the development of optical coherence tomography. In this application, either $\mathrm{cw}$ or greater than kilohertz pulsed signals are required for sample scanning. Although light generation based on $\chi^{(2)}$ nonlinear optics has not been used for this purpose, its potential deserves further exploration.
Retracing behavior (RB) of a phase-matching curve (output wavelength relative to a phase-matching angle or another parameter) was well studied in birefringence phase matching (BPM) ${ }^{5-8}$ RB implies that a phasematched signal or idler wavelength is not a monotonically increasing or decreasing function of crystal orientation angle or another crystal-related parameter. In such a situation, more than one signal-idler set in a parametric process can be simultaneously phase matched. Also, the shape variation of a phase-matching curve usually results in an almost vertical curve under certain conditions, possibly leading to broadband parametric processes. Although broadband generation based on $\chi^{(2)}$ nonlinear optics can be implemented near the degenerate points in optical parametric processes, ${ }^{9,10}$ its applicable ranges are limited. In particular, the signal and idler contributions to a broadband output usually propagate in different directions, leading to difficulty in application. With $\mathrm{RB}$ used in various nonlinear crystals with either collinear or noncollinear BPM, broadband generation of more applicable ranges has been reported. ${ }^{5-8}$ Usually the availability of broadband generation with collinear phase matching is limited. With noncollinear phase matching, a larger variety of broadband operations is available. Experimentally, multiple-wavelength and broadband outputs based on RB in either a collinear or a noncollinear BPM scheme have been reported. ${ }^{7,8}$

Although QPM in the collinear configuration can increase interaction length without the problem of walk-off, some reports have demonstrated the advantages of an angle-tuned optical parametric oscillator (OPO) with periodically poled $\mathrm{LiNbO}_{3}$ (PPLN; Refs. 11 and 12) and- 
$\mathrm{KTiOPO}_{4}$ (KTP; Ref. 13) based on noncollinear interaction. Meanwhile, similarly to the BPM case, broadband generation with collinear QPM can be implemented near the degenerate point. Engineering quasi-phase-matched structures will make broadband generation also feasible. ${ }^{14}$ In this paper we report systematic theoretical results of broadband operation based on $\mathrm{RB}$ with collinear, noncollinear, and quasi-collinear phase matching in four commonly used quasi-phase-matched crystals, including PPLN, periodically poled KTP (PPKTP), periodically poled $\mathrm{KTiOAsO}_{4}$ (PPKTA), and periodically poled $\mathrm{LiTaO}_{3}$ (PPLT). Although noncollinear pumping of quasi-phase-matched nonlinear processes has been reported, ${ }^{11-13}$ we have not seen $\mathrm{RB}$ for broadband generation discussed in the literature.

This paper is organized as follows: In Section 2 the phase-matching conditions for solving for $\mathrm{RB}$ are discussed. Conditions for and results of broadband operations based on RB with collinear, noncollinear, and quasicollinear phase matching in various QPM crystals are presented in Sections 3, 4, and 5, respectively. Typically, we prefer operation of the device at room temperature. Room-temperature operation is usually not difficult for crystals such as KTP, KTA and LT. However, because photoinduced damage will occur in $\mathrm{LiNbO}_{3}$ with relatively high pump power, PPLN devices are usually operated at temperatures higher than $150{ }^{\circ} \mathrm{C}$. Therefore, in discussing RB and broadband generation in PPKTP, PPKTA, and PPLT we consider operation at room temperature, whereas temperatures of $150^{\circ} \mathrm{C}$ or higher are assumed for PPLN. Discussions of a few general rules for finding broadband operation, and our conclusions, are given in Section 6.

\section{THEORETICAL ANALYSIS}

In this section we present the theoretical formulations for $\mathrm{RB}$ calculations in quasi-phase-matched crystals. We pay particular attention to noncollinear phase matching configurations. Figure 1 shows the orientations of various interacting waves in a quasi-phase-matched crystal with a noncollinear phase-matching configuration. Here $\mathbf{K}_{p}$ represents the wave vector of the pump beam, which

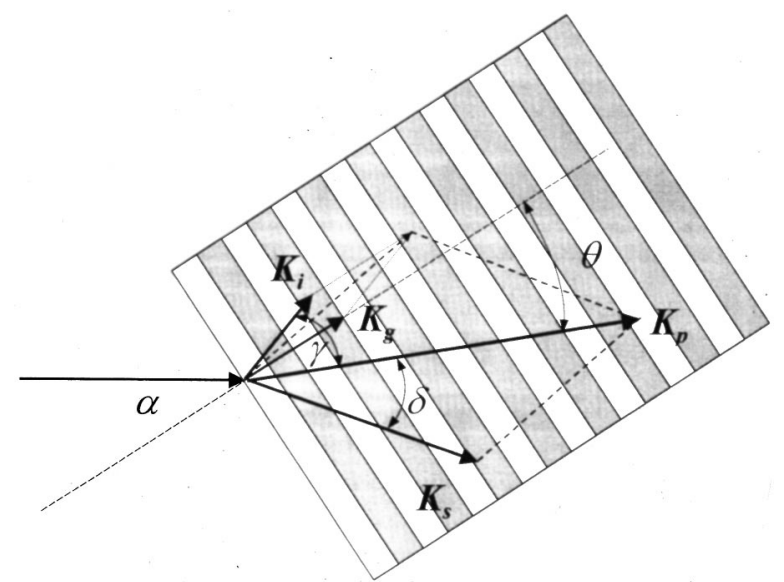

Fig. 1. Orientations of various interacting wave vectors in a quasi-phase matched crystal with a noncollinear phase-matching configuration. lies at an angle $\theta$ from the QPM axis, i.e., in the direction of the QPM wave vector $\mathbf{K}_{g} . \mathbf{K}_{s}$ and $\mathbf{K}_{i}$, which are the wave vectors of the signal and the idler, respectively, lie at angles $\delta$ and $\gamma$ with respect to $\mathbf{K}_{p}$. Angle $\alpha$ is the incident angle of the pump in air with respect to the QPM axis. For the noncollinear phase matching shown in Fig. 1 the phase-matching conditions are described by the following two equations:

$$
\begin{aligned}
& \frac{n\left(\lambda_{s}, T\right)}{\lambda_{s}} \sin \delta+\frac{n\left(\lambda_{i}, T\right)}{\lambda_{i}} \sin \gamma \\
&+\frac{m}{\Gamma(T)} \sin \theta=0, \\
& \frac{n\left(\lambda_{s}, T\right)}{\lambda_{s}} \cos \delta+\frac{n\left(\lambda_{i}, T\right)}{\lambda_{i}} \cos \gamma \\
&+\frac{m}{\Gamma(T)} \cos \theta=\frac{n\left(\lambda_{p}, T\right)}{\lambda_{p}} .
\end{aligned}
$$

Here $\lambda_{p}, \lambda_{s}$, and $\lambda_{i}$ are the wavelengths of the pump, the signal, and the idler, respectively. Throughout the entire paper we assume a wavelength of the signal shorter than that of the idler. $n$, which depends on wavelength $\lambda$ and temperature $T$, is the material's refractive index. ${ }^{15-18}$ Parameter $\Gamma$ represents the period of domain inversion or $\chi^{(2)}$ coefficient distribution of the nonlinear crystal. Because of thermal expansion, $\Gamma$ is dependent on temperature $T$; nevertheless, the dependence is usually weak. Also, integer $m$ stands for the order of QPM used for phase matching. Note that, when $\theta=\delta=\gamma=0$, a collinear phase-matching configuration results. Equations (1) and (2) are to be solved for various conditions. We assume that $m=1$ for the first-order QPM in this paper.

\section{RETRACING BEHAVIORS AND BROADBAND GENERATION WITH COLLINEAR PHASE MATCHING}

$\mathrm{RB}$ can be observed with the collinear phase-matching configuration in quasi-phase-matched crystals. Figure 2 shows the collinear phase-matching curves relative to the QPM period of PPLN for various pump wavelengths ranging from 500 to $1000 \mathrm{~nm}$ with the temperature fixed at $150{ }^{\circ} \mathrm{C}$. We can observe $\mathrm{RB}$ of the phase-matching curve when the pump wavelength is $650-950 \mathrm{~nm}$. One can see that, as the pump wavelength increases, the phasematching curve changes its shape. This implies that at a certain pump wavelength the tuning curve is retroflected, which results in an almost vertical phase-matching curve. Almost vertical phase-matching curves can be seen with the pump wavelength near $950 \mathrm{~nm}$ (Fig. 2). Such phenomena are illustrated on a finer scale in Fig. 3. With the pump at $960 \mathrm{~nm}$ and the PPLN period at $27.5 \mu \mathrm{m}$, a vertical phase-matching curve from 1.68 to $2.24 \mu \mathrm{m}$ can clearly be seen (as is also apparent from the first row of Table 1). This pump wavelength is defined as $\lambda_{\mathrm{BC}}$. This wavelength becomes a key parameter in describing $\mathrm{RB}$ with noncollinear phase-matching configurations, as we discuss in Section 4. A vertical phase-matching curve implies the simultaneous generation of a broadband source. However, such broadband operation can occur 
only near the degenerate point when the pump wavelength is longer than $900 \mathrm{~nm}$. When the broadband wavelength is greater than $1600 \mathrm{~nm}$, the configuration is not suitable for fiber communication applications.

RB for collinear quasi-phase-matched optical parametric processes can also be observed in other materials, such

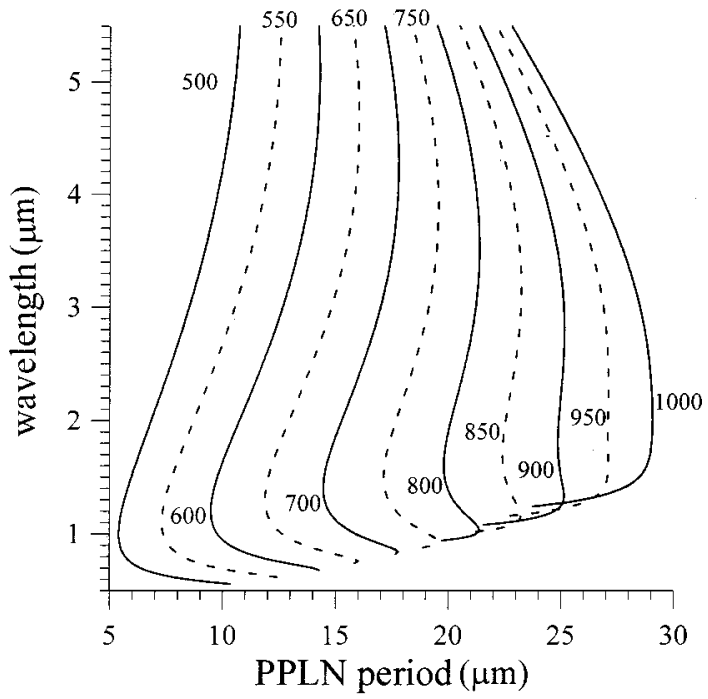

Fig. 2. Phase-matched wavelength versus the QPM period of PPLN in the collinear phase-matching scheme for pump wavelengths ranging from 500 to $1000 \mathrm{~nm}$ with temperature fixed at $150{ }^{\circ} \mathrm{C}$

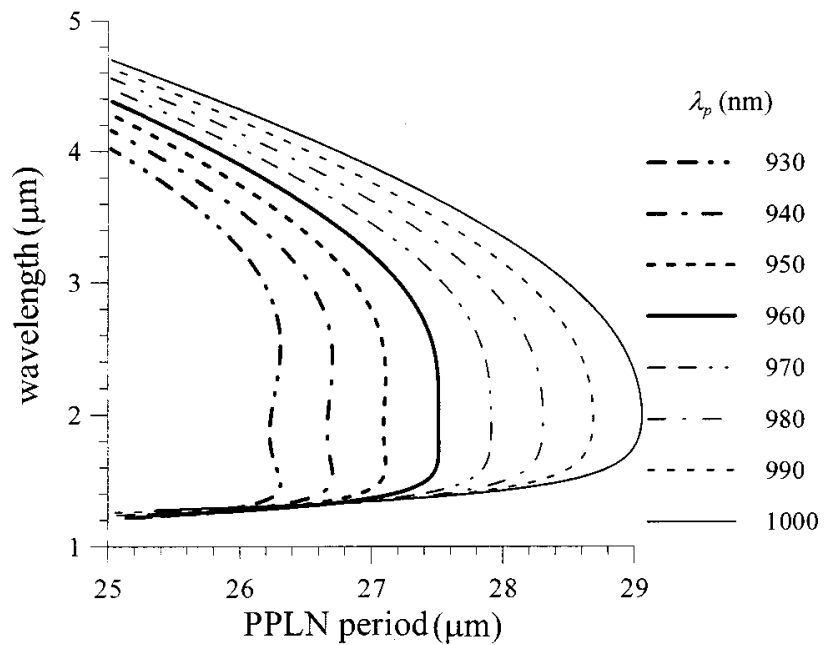

Fig. 3. Phase-matched wavelength versus QPM period of PPLN in the collinear phase-matching scheme for pump wavelengths ranging from 930 to $1000 \mathrm{~nm}$ with temperature fixed at $150^{\circ} \mathrm{C}$.

Table 1. Broadband Operation with Various Phase-Matching Methods Based on PPLN

\begin{tabular}{llccc}
\hline $\begin{array}{c}\text { Type of phase } \\
\text { Matching }\end{array}$ & $\lambda_{p}(\mathrm{~nm})$ & $\begin{array}{c}\text { PPLN Period } \\
(\mu \mathrm{m})\end{array}$ & $\lambda_{s}(\mu \mathrm{m})$ & $\theta\left(^{\circ}\right)$ \\
\hline $\begin{array}{l}\text { Collinear } \\
\left(150{ }^{\circ} \mathrm{C}\right)\end{array}$ & $960\left(\lambda_{\mathrm{BC}}\right)$ & 27.5 & $1.68-2.24$ & 0 \\
Noncollinear & 905 & 24.5 & $1.45-1.65$ & 1.2 \\
$\quad\left(180^{\circ} \mathrm{C}\right)$ & 780 & 17.4 & $1.198-1.298$ & 2.594 \\
$\begin{array}{l}\text { Quasi-collinear } \\
\left(180^{\circ} \mathrm{C}\right)\end{array}$ & 904 & 15.2 & $1.45-1.65$ & 51.046 \\
\hline
\end{tabular}

Table 2. Broadband Operation with Various Phase-Matching Methods Based on PPKTP

\begin{tabular}{llccc}
\hline $\begin{array}{c}\text { Type of Phase } \\
\text { Matching }\end{array}$ & $\lambda_{p}(\mathrm{~nm})$ & $\begin{array}{c}\text { PPKTP Period } \\
(\mu \mathrm{m})\end{array}$ & $\lambda_{s}(\mu \mathrm{m})$ & $\theta\left(^{\circ}\right)$ \\
\hline $\begin{array}{l}\text { Collinear } \\
\left(27^{\circ} \mathrm{C}\right)\end{array}$ & $900\left(\lambda_{\mathrm{BC}}\right)$ & 32.3 & $1.61-2.05$ & 0 \\
Noncollinear & 860 & 29.8 & $1.435-1.6$ & 0.96 \\
$\quad\left(27^{\circ} \mathrm{C}\right)$ & 750 & 21.8 & $1.15-1.27$ & 2.311 \\
Quasi-collinear & 870 & 22.6 & $1.46-1.6$ & 41.72 \\
$\left(27^{\circ} \mathrm{C}\right)$ & 750 & 9 & $1.16-1.24$ & 64.425 \\
\hline
\end{tabular}

Table 3. Broadband Operation with Various Phase-Matching Methods Based on PPKTA

\begin{tabular}{llccc}
\hline $\begin{array}{l}\text { Type of Phase } \\
\text { Matching }\end{array}$ & $\lambda_{p}(\mathrm{~nm})$ & $\begin{array}{c}\text { PPKTP Period } \\
(\mu \mathrm{m})\end{array}$ & $\lambda_{s}(\mu \mathrm{m})$ & $\theta\left({ }^{\circ}\right)$ \\
\hline $\begin{array}{l}\text { Collinear } \\
\left(27^{\circ} \mathrm{C}\right)\end{array}$ & $980\left(\lambda_{\mathrm{BC}}\right)$ & 37.7 & $1.66-2.4$ & 0 \\
Noncollinear & 940 & 35.2 & $1.53-1.81$ & 0.78 \\
$\left(27^{\circ} \mathrm{C}\right)$ & 780 & 23.1 & $1.18-1.31$ & 2.51 \\
Quasi-collinear & 940 & 24.4 & $1.53-1.82$ & 45.6 \\
$\left(27^{\circ} \mathrm{C}\right)$ & 780 & 8.6 & $1.12-1.3$ & 66.8 \\
\hline
\end{tabular}

Table 4. Broadband Operation with Various Phase-Matching Methods Based on PPLT

\begin{tabular}{llccc}
\hline $\begin{array}{l}\text { Type of Phase } \\
\text { Matching }\end{array}$ & $\lambda_{p}(\mathrm{~nm})$ & $\begin{array}{c}\text { PPLT Period } \\
(\mu \mathrm{m})\end{array}$ & $\lambda_{s}(\mu \mathrm{m})$ & $\theta\left(^{\circ}\right)$ \\
\hline $\begin{array}{l}\text { Collinear } \\
\left(27^{\circ} \mathrm{C}\right)\end{array}$ & $910\left(\lambda_{\mathrm{BC}}\right)$ & 27.27 & $1.6-2.1$ & 0 \\
Noncollinear & 880 & 25.75 & $1.468-1.638$ & 0.79 \\
$\quad\left(27^{\circ} \mathrm{C}\right)$ & 750 & 18.2 & $1.155-1.245$ & 2.393 \\
Quasi-collinear & 880 & 19.3 & $1.468-1.628$ & 41.044 \\
$\left(27^{\circ} \mathrm{C}\right)$ & 750 & 7.5 & $1.155-1.235$ & 64.479 \\
\hline
\end{tabular}

as PPKTP, PPKTA, and PPLT. Broadband operations are also feasible in PPKTP, PPKTA, and PPLT. Pump wavelength $\lambda_{\mathrm{BC}}$ for observing broadband operation in collinear phase matching with PPKTP, PPKTA, and PPLT is 900,980 , and $910 \mathrm{~nm}$, respectively, when the temperatures of the compounds are fixed at $27^{\circ} \mathrm{C}$. Again, broadband operation is feasible only near the degenerate point. The corresponding quasi-phase-matched periods and the resultant signal wavelength ranges are listed in the first rows of Tables 2-4. Note that the pump wavelength for observing a vertical phase-matching curve in the collinear configuration and hence the resultant broadband range might vary slightly with crystal temperature. However, under collinear phase-matching conditions the variation is quite limited. In all four quasi-phase-matched crystals with which we are concerned, $\lambda_{\mathrm{BC}}$ values are always larger than $900 \mathrm{~nm}$, and the resultant broadband outputs are outside the fiber communication wavelength range.

\section{RETRACING BEHAVIORS AND BROADBAND GENERATION WITH NONCOLLINEAR PHASE MATCHING}

In general, the maximum nonlinearity of quasi-phasematched material is $d_{33}$ (for PPLN, PPKTP, PPKTA, and 
PPLT). The collinear quasi-phase-matched configuration has the advantages of a large nonlinear coefficient and no walk-off. Therefore the crystal length can be as large as possible for efficient operation. ${ }^{19}$ Nevertheless, a noncollinear configuration provides an extra parameter (the angle) for tuning a quasi-phase-matched OPO. ${ }^{11-13}$ Also, the variation of this extra parameter results in $\mathrm{RB}$. Because there are many variable parameters, certain constraints are required for simplifying the vector relationship described in Fig. 1 and by Eq. (1) and (2). In the following discussions we shall always consider the operation of an OPO in which either a resonating signal or a resonating idler wave vector is defined to be parallel to the QPM axis. In other words, $\delta, \gamma=\theta$. In this situation we can vary the pump's incident angle $(\theta)$ for various phase-matching conditions.

Figures 4, 5, and 6 show the tuning curves of a noncollinear signal-resonated PPLN OPO with pump wavelengths at 600,800 and $1000 \mathrm{~nm}$, respectively, for several QPM periods. The PPLN temperature was fixed at $160^{\circ} \mathrm{C}$. With QPM, a phase-matching condition can be achieved only in a certain range of periods for a particular pump wavelength. Here we can observe RB with certain PPLN periods. For instance, RB can be observed with a period ranging from 18.8 to $20 \mu \mathrm{m}$ in an 800-nm-pumped signal-resonated PPLN OPO. We did not observe RB in a signal-resonated OPO with pump wavelength longer than a value that was shorter than $1000 \mathrm{~nm}$, as shown in Fig. 6. Nevertheless, RB can be observed in idler-resonated PPLN OPO with longer pump wavelengths, as shown in Fig. 7, which was obtained with the same pump wavelength $(1000 \mathrm{~nm})$ as was Fig. 6 . It is interesting to find that, in a signal-resonated OPO with a noncollinear configuration, RB can be observed only for pump wavelengths shorter than that which leads to a vertical phasematching curve or to broadband operation in a collinear configuration, i.e., $\lambda_{\mathrm{BC}}$. Also, in an idler-resonated OPO with a noncollinear configuration $\mathrm{RB}$ can be observed only for pump wavelengths longer than $\lambda_{\mathrm{BC}}$. This rule seems quite general for various quasi-phase-matched crystals and even for the BPM. ${ }^{8}$

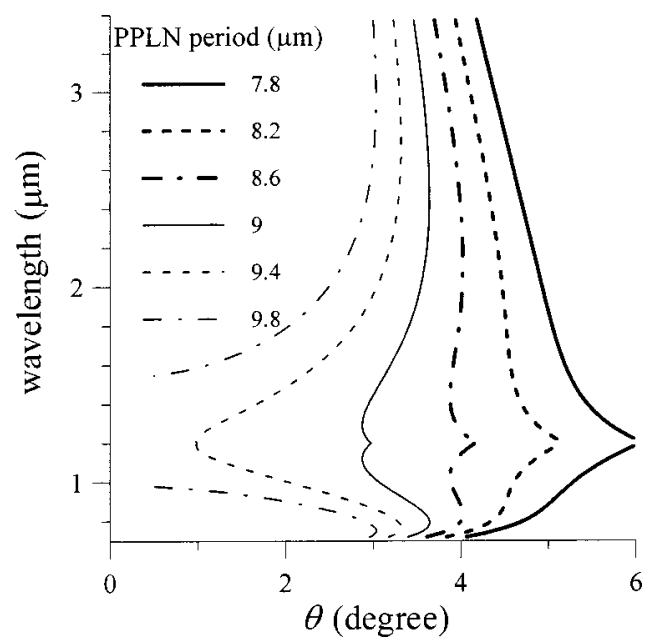

Fig. 4. Tuning curves of a noncollinear signal-resonated PPLN OPO with pump wavelength at $600 \mathrm{~nm}$ and temperature fixed at $160{ }^{\circ} \mathrm{C}$ for several QPM periods.

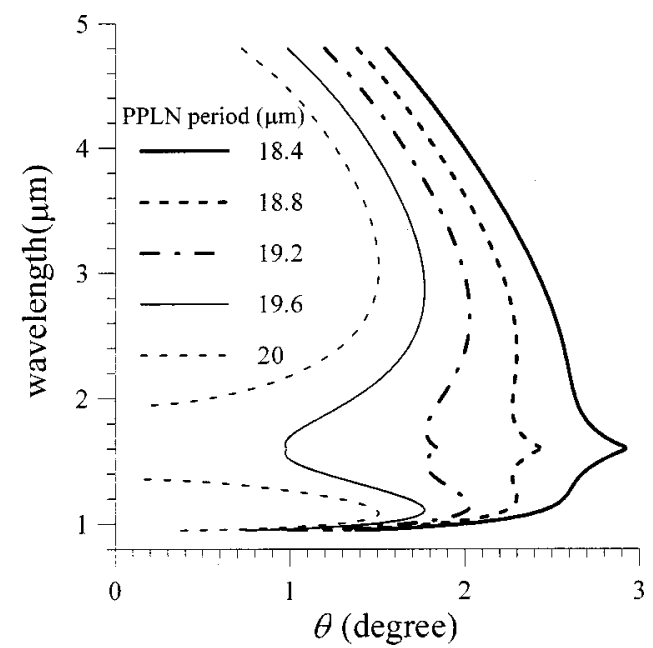

Fig. 5. Tuning curves of a noncollinear signal-resonated PPLN OPO with pump wavelength at $800 \mathrm{~nm}$ and temperature fixed at $160{ }^{\circ} \mathrm{C}$ for several QPM periods.

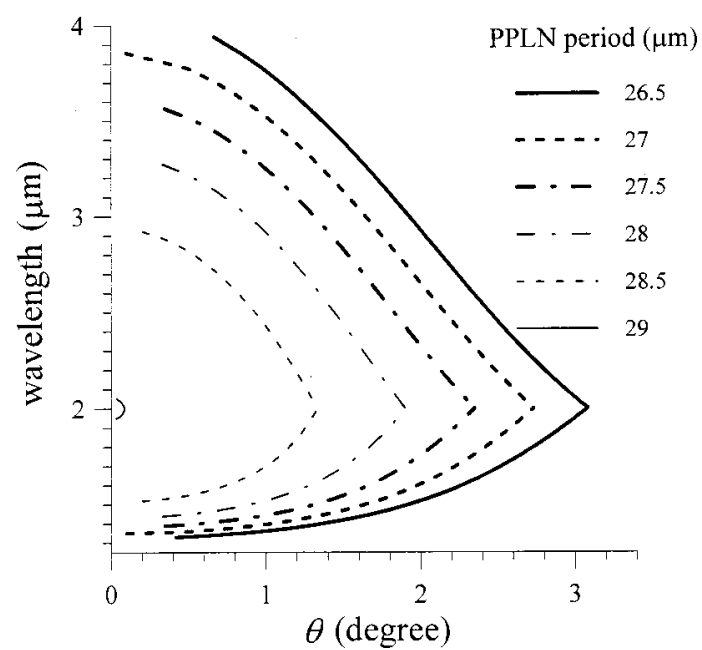

Fig. 6. Tuning curves of a noncollinear signal-resonated PPLN OPO with pump wavelength at $1000 \mathrm{~nm}$ and temperature fixed at $160{ }^{\circ} \mathrm{C}$ for several QPM periods.

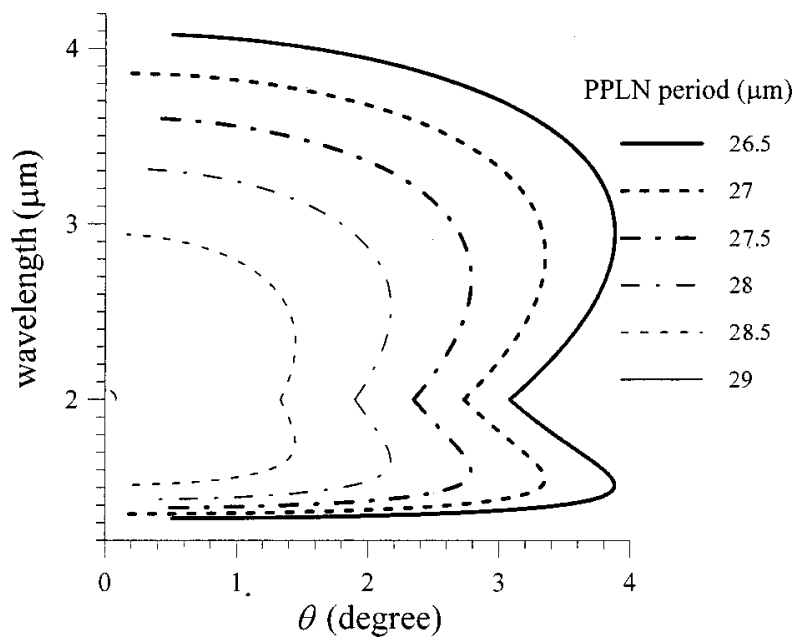

Fig. 7. Tuning curves of a noncollinear idler-resonated PPLN OPO with pump wavelength at $1000 \mathrm{~nm}$ and temperature fixed at $160^{\circ} \mathrm{C}$ for several QPM periods. 


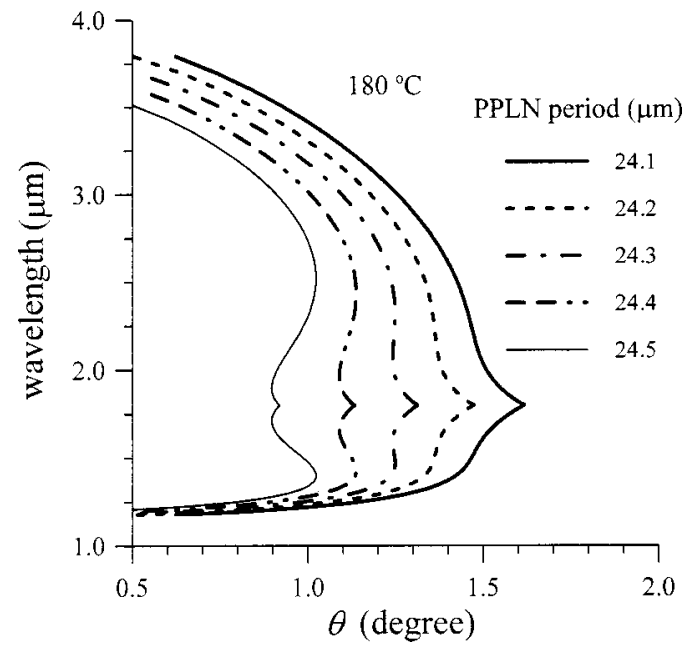

Fig. 8. Tuning curves of a noncollinear, signal-resonated PPLN OPO with pump wavelength at $900 \mathrm{~nm}$. Broadband operation appears when the PPLN period is near $24.3 \mu \mathrm{m}$.

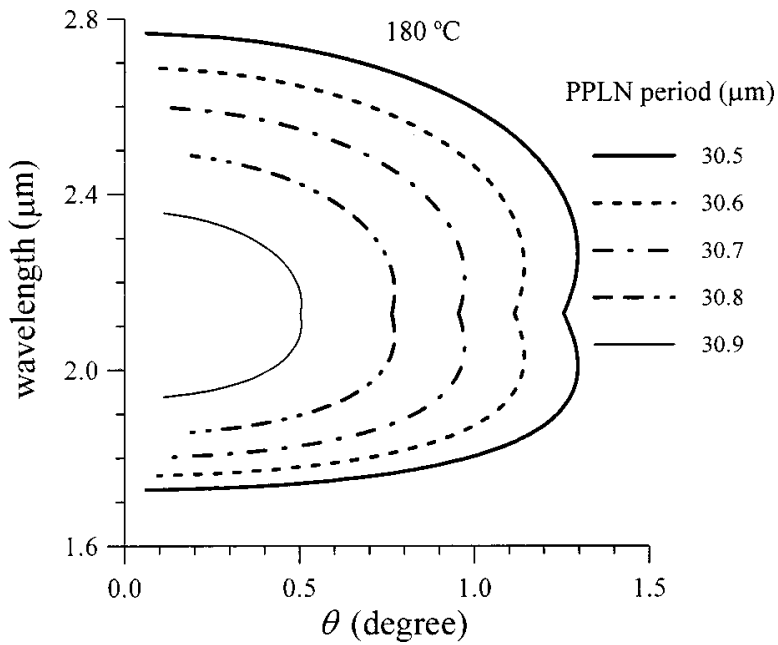

Fig. 9. Tuning curves of a noncollinear, idler-resonated PPLN OPO with pump wavelength at $1064 \mathrm{~nm}$. Broadband operation appears when the PPLN period is near $30.7 \mu \mathrm{m}$.

Broadband operation is feasible when $\mathrm{RB}$ can be observed. It was found that broadband operation for an idler-resonated OPO with pump wavelength longer than $\lambda_{\mathrm{BC}}$ can be obtained only at degenerate points. However, broadband phenomena for a signal-resonated OPO with a pump wavelength shorter than $\lambda_{\mathrm{BC}}$ can be observed either at degenerate points or in signal wavelength ranges away from the degenerate points. Figures 8 and 9 show the noncollinear phase-matching curves for a signalresonated PPLN OPO with a pump wavelength at $900 \mathrm{~nm}$ and for an idler-resonated PPLN OPO pumped at 1064 $\mathrm{nm}$, respectively. The PPLN temperature for these two cases was fixed at $180^{\circ} \mathrm{C}$. In Fig. 8 one can see a vertical curve for a signal near $1550 \mathrm{~nm}$ and an idler near $2.25 \mu \mathrm{m}$ when the PPLN period is $24.3 \mu \mathrm{m}$ and $\theta$ is $\sim 1.24^{\circ}$. In Fig. 9 there are almost vertical phase-matching curves near the degenerate point. Such results are useful for wavelength conversion in fiber communications. ${ }^{3}$ Unfortunately, the wavelength range is not within a useful window. In Table 1 are listed two sets of broadband data for a noncollinear phase-matched signal-resonated PPLN OPO. One set is good for signal amplification in fiber communication applications. The other is useful for optical coherence tomography applications.

Similar RB and broadband operation exists in other quasi-phase-matched crystals with noncollinear phase matching. Two sets of broadband data for PPKTP, PPKTA, and PPLT are given in Tables 2, 3, and 4, respectively. The conditions of broadband operation in PPKTP, PPKTA, and PPLT are the same as for PPLN, as described above.

\section{RETRACING BEHAVIOR AND BROADBAND GENERATION WITH QUASI- COLLINEAR PHASE MATCHING}

Next, we consider a quasi-collinear configuration, as shown in Fig. 10. In this configuration, two of the three interacting waves are parallel. Here we assume that the direction of signal propagation is parallel to that of the pump ( $\delta=0$ ) for signal resonance. Figures 11 and 12 show the tuning curves of a quasi-collinear signalresonated PPLN OPO with pump wavelengths at 800 and $900 \mathrm{~nm}$, respectively, for several corresponding periods. The crystal temperature was $180^{\circ} \mathrm{C}$. We can observe $\mathrm{RB}$ in certain ranges of QPM periods. The major advantage of the quasi-collinear configuration is that pump and signal propagate together and hence the interaction length can be increased (compared with that in the noncollinear case). This is particularly useful in a waveguide structure. The major disadvantage of this method is that phase-matching angle $\theta$ usually must be large for RB to be observed. Because the refractive index of PPLN is quite large (near 2.2), the critical angle for total internal reflection is small (below $30^{\circ}$ ). Therefore we may need a wedge-cut PPLN for coupling waves into and out of the crystal.

Broadband operation with quasi-collinear configurations was shown in Figs. 11 and 12. Figure 13 shows optimum broadband conditions for amplifying fiber communication signals. In this quasi-collinear case, PPLN with a $15.2-\mu \mathrm{m}$ period is pumped with wavelengths near 900 $\mathrm{nm}$. When the pump wavelength is $904 \mathrm{~nm}$ and phasematched angle $\theta$ is $51.046^{\circ}$, an almost vertical phase-

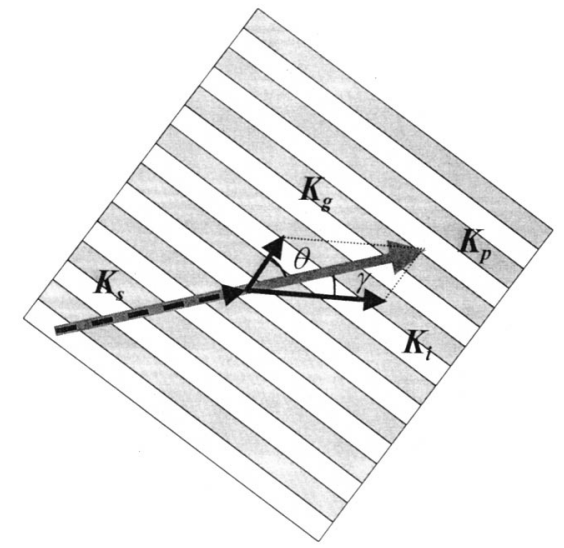

Fig. 10. Orientations of various interacting wave vectors in a quasi-phase-matched crystal with a quasi-collinear phasematching configuration. 


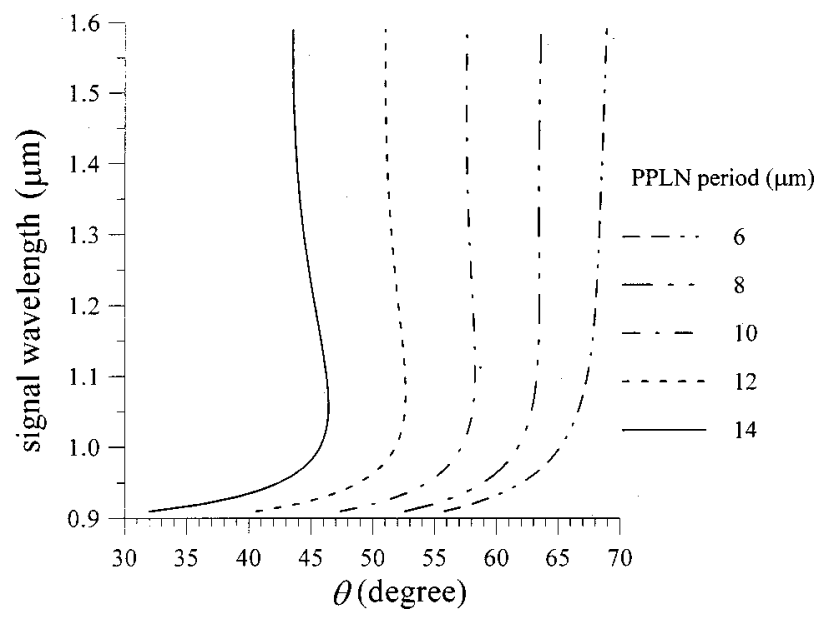

Fig. 11. Tuning curves of quasi-collinear, signal-resonated PPLN OPO with pump wavelength at $800 \mathrm{~nm}$ and crystal temperature fixed at $180{ }^{\circ} \mathrm{C}$.

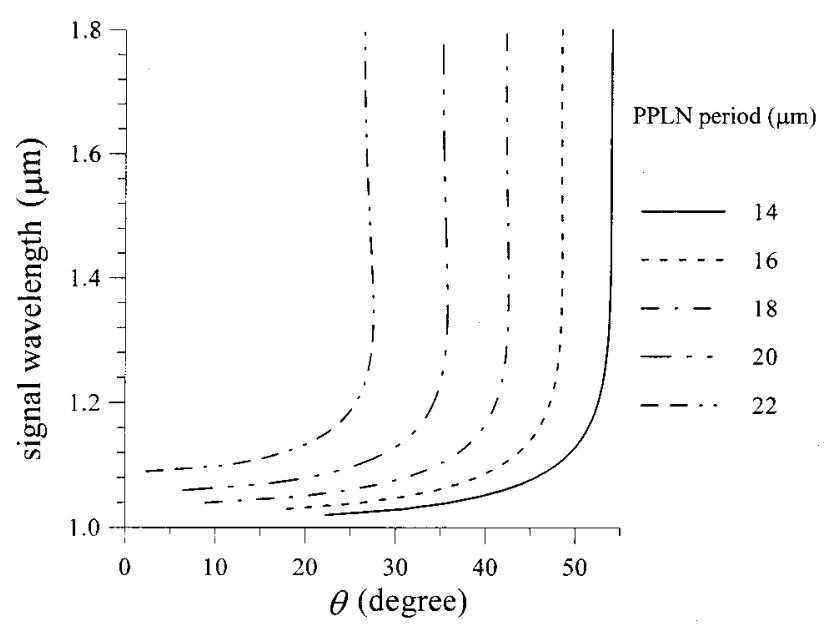

Fig. 12. Tuning curves of quasi-collinear, signal-resonated PPLN OPO with pump wavelength at $900 \mathrm{~nm}$ and crystal temperature fixed at $180^{\circ} \mathrm{C}$.

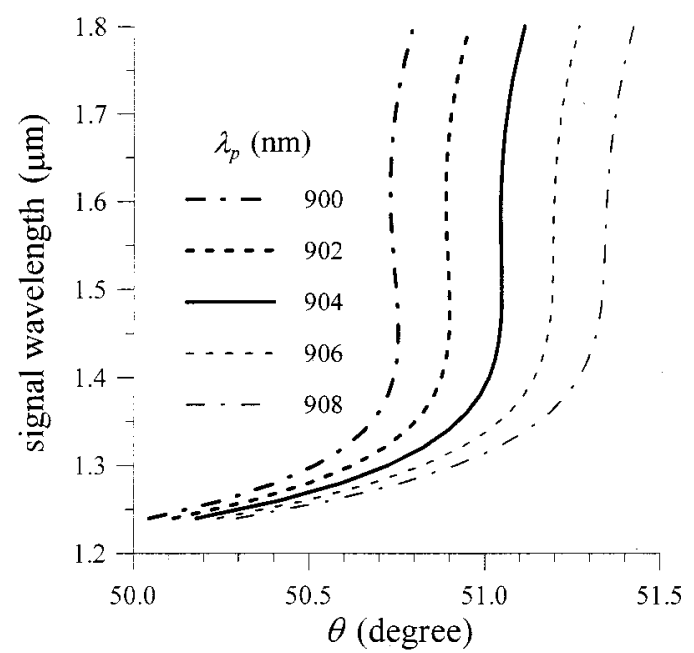

Fig. 13. Tuning curves of a quasi-collinear, signal-resonated PPLN OPO with PPLN period fixed at $15.2 \mu \mathrm{m}$ and crystal temperature fixed at $180^{\circ} \mathrm{C}$. Broadband operation is feasible with pump wavelength at $904 \mathrm{~nm}$.

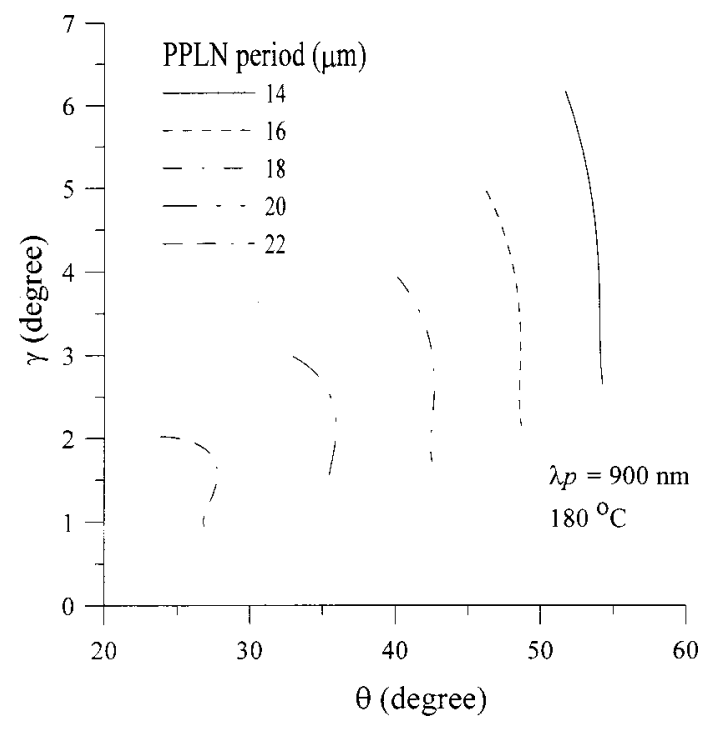

Fig. 14. $\quad \gamma$ angle values versus $\theta$ angle values for the five curves in Fig. 12.

matching curve from 1450 to $1650 \mathrm{~nm}$ can be obtained. The corresponding idler wavelength ranges from 2 to 2.4 $\mu \mathrm{m}$. Figure 14 shows the variation of angle $\gamma$ with $\theta$ for the five curves in Fig. 12. One can see that the direction of idler propagation deviates from that of the pump by $1^{\circ}-6^{\circ}$ inside the crystal. By using such a quasi-collinear configuration we can achieve signal amplification for broadband wavelength-division multiplexing fiber communication with a single pump wave. In Table 1, two sets of broadband data with quasi-collinear phase matching in PPLN are compared. Broadband operation for a quasi-collinear QPM OPO can also be observed in PPKTP, PPKTA, and PPLT. Conditions for each type of crystal are shown in Tables 2,3 , and 4 , respectively.

\section{DISCUSSIONS AND CONCLUSIONS}

As we mentioned above, in a signal-resonated (idlerresonated) $\mathrm{OPO}$ with a noncollinear configuration $\mathrm{RB}$ and hence broadband operation can be observed only for pump wavelengths shorter (longer) than $\lambda_{\mathrm{BC}}$. Also, broadband operation for an idler-resonated OPO with a pump wavelength longer than $\lambda_{\mathrm{BC}}$ can be obtained only at degenerate points. Nevertheless, broadband phenomena for a signal-resonated OPO with a pump wavelength shorter than $\lambda_{\mathrm{BC}}$ can be observed either at or away from the degenerate points. These rules are also true for quasicollinear configurations. They are actually quite general for all concerned quasi-phase-matched crystals and are valid for the BPM configuration.

Besides these trends, a few common phenomena for broadband operation based on quasi-phase-matched crystals deserve mention. First, as the pump wavelength approaches $\lambda_{\mathrm{BC}}$, the phase-matching angle for implementing a broadband OPO becomes smaller (closer to the collinear case). In this situation the broadband signal wavelength range is closer to the degenerate point and the signal bandwidth becomes larger. Second, the phasematched QPM period for a broadband OPO in a quasicollinear configuration is always smaller than that in a 
noncollinear scheme. With a shorter pump wavelength, the difference in QPM period becomes larger. Third, the phase-matching angles for a broadband OPO in the quasicollinear case become quite large, particularly with short pump wavelengths. With a shorter pump wavelength, the angle between idler and pump becomes larger. In this situation the overall efficiency will be reduced.

For noncollinear and quasi-collinear configurations, broadband generation can be obtained at the expense of nonlinear conversion efficiency. In both cases the advantage of increasing crystal length for enhancing conversion efficiency in the collinear configuration does not exist. Considering the operation of difference-frequency generation, the conversion efficiency depends on the degree of overlap of the incident pump and signal-idler beams. For the quasi-collinear case, if a waveguide structure is fabricated, the conversion efficiency is actually not degraded compared with that of a typical collinear configuration with a waveguide structure. This is so because usually the fabricated waveguide cannot guide the idler; the result is a situation similar to that for the quasicollinear configuration.

From the results reported above, one can see that the suitable pump wavelengths for broadband generation are always shorter than $900 \mathrm{~nm}$. Such high-coherence pump sources are available commercially. For instance, a widely tunable narrow-bandwidth Ti:sapphire laser (with a ring cavity) should be useful for such operations. When a compact setup is required, such as for the application to fiber communications, highly coherent laser diodes based on GaAs/AlGaAs or GaAs/InGaAs quantum-well structures should be developed first.

In summary, we have numerically demonstrated the retracing behavior of a quasi phase-matching curve, which is the key phenomenon for broadband optical parametric processes. With QPM, vertical phase-matching curves and hence broadband operations exist in collinear, noncollinear, and quasi-collinear configurations. Broadband operation is feasible only at the degenerate point in the collinear configuration with a specific pump wavelength, $\lambda_{\mathrm{BC}}$. Such a constraint sets a significant limitation on applications. In particular, all the broadband signals of collinear phase-matched PPLN, PPKTP, PPKTA, PPLT, and quasi-phase-matched GaAs (the data are not discussed in this paper) are beyond the fiber communication wavelength range and biological window (650-1300 nm). With a noncollinear or quasi-collinear phase-matching configuration the conditions and output signal ranges of broadband operation are much more flexible, particularly those that are useful for fiber communication and optical imaging of biological tissues.

\section{ACKNOWLEDGMENTS}

This research was supported by the National Science Council, The Republic of China, under the grants NSC 892218-E-002-094, NSC 89-2218-E-002-095, and NSC 892215-E-002-051.

\section{C.-C. Yang's e-mail address is ccy@cc.ee.ntu.edu.tw.}

\section{REFERENCES}

1. A. Galvanauskas, K. K. Wong, K. El Hadi, M. Hofer, M. E. Fermann, D. Harter, M. H. Chou, and M. M. Fejer, "Amplification in 1.2-1.7 $\mu \mathrm{m}$ communication window using OPA in PPLN waveguides," Electron. Lett. 35, 731-732 (1999).

2. M. H. Chou, I. Brener, K. R. Parameswaran, and M. M. Fejer, "Stability and bandwidth enhancement of difference frequency generation (DFG)-based wavelength conversion by pump detuning," Electron. Lett. 35, 978-979 (1999).

3. M. H. Chou, I. Brener, M. M. Fejer, E. E. Chaban, and S. B. Christman, "1.5- $\mu \mathrm{m}$-band wavelength conversion based on cascaded second-order nonlinearity in $\mathrm{LiNbO}_{3}$ waveguides," IEEE Photon. Technol. Lett. 11, 653-655 (1999).

4. W. Drexler, U. Morgner, F. X. Kartner, C. Pitris, S. A. Boppart, X. D. Li, E. P. Ippen, and J. G. Fujimoto, "In vivo ultrahigh-resolution optical coherence tomography," Opt. Lett. 24, 1221-1223 (1999).

5. S. Lin, B. Wu, F. Xie, and C. Chen, "Phase-matching retracing behavior: new features in $\mathrm{LiB}_{3} \mathrm{O}_{5}$," Appl. Phys. Lett. 59, 1541-1543 (1991).

6. X. Liu, D. Deng, M. Li, D. Guo, and Z. Xu, "Retracing behavior of the phase-matching angle of nonlinear crystals in optical parametric oscillators," J. Appl. Phys. 74, 29892991 (1993).

7. J. Wang, M. H. Dunn, and C. F. Rae, "Polychromatic optical parametric generation by simultaneous phase matching over a large spectral bandwidth," Opt. Lett. 22, 763-765 (1997).

8. S. D. Huang, C. W. Hsu, D. W. Huang, and C. C. Yang, "Retracing behaviors of the phase-matching angle in noncollinear phase-matched optical parametric oscillators," J. Opt. Soc. Am. B 15, 1375-1380 (1998).

9. A. J. Campillo, R. C. Hyer, and S. L. Shapiro, "Picosecond infrared-continuum generation by three-phonon parametric amplification in $\mathrm{LiNbO}_{3}$," Opt. Lett. 4, 357-359 (1979).

10. A. Birmontas, A. Piskarskas, and A. Stabinis, "Dispersion anomalies of tuning characteristics and spectrum of an optical parametric oscillator," Sov. J. Quantum Electron. 13, 1243-1245 (1983).

11. S. T. Yang and S. P. Velsko, "Frequency-agile kilohertz repetition-rate optical parametric oscillator based on periodically poled lithium niobate," Opt. Lett. 24, 133-135 (1999).

12. M. J. Missey, V. Dominic, P. E. Powers, and K. L. Schepler, "Periodically poled lithium niobate monolithic nanosecond optical parametric oscillators and generators," Opt. Lett. 24, 1227-1229 (1999).

13. V. Smilgevicius, A. Stabinis, A. Piskarskas, V. Pasiskevicius, J. Hellstrom, S. Wang, and F. Laurell, "Non-collinear optical parametric oscillator with periodically poled KTP," Opt. Commun. 173, 365-369 (2000).

14. S. M. Russell, M. J. Missey, P. E. Powers, and K. L. Schepler, "Broadband mid-infrared generation in elliptically pumped periodically poled lithium niobate devices," in Conference on Lasers and Electro-Optics (CLEO), Vol. 56 of OSA Trends in Optics and Photonics Series (Optical Society of America, Washington, D.C., 2001), paper CThJ4.

15. V. G. Dmitriev, G. G. Gurzdyan, and D. N. Nikogosyan, Handbook of Nonlinear Optical Crystals (Springer-Verlag, Berlin, 1991).

16. J. P. Meyn and M. M. Fejer, "Tunable ultraviolet radiation by second-harmonic generation in periodically poled lithium tantalate," Opt. Lett. 22, 1214-1216 (1997).

17. K. Fradkin, A. Arie, A. Skilar, and G. Rosenman, "Tunable mid-infrared source by difference frequency generation in bulk periodically poled $\mathrm{KTiOPO}_{4}$," Appl. Phys. Lett. 74, 914-916 (1999).

18. K. Fradkin-Kashi, A. Arie, P. Urenski, and G. Rosenman, "Mid-infrared difference-frequency generation in periodically poled $\mathrm{KTiOAsO}_{4}$ and application to gas sensing," Opt. Lett. 25, 743-745 (2000).

19. G. D. Miller, R. G. Batchko, W. M. Tulloch, D. R. Weise, M. M. Fejer, and R. L. Byer, " $42 \%$-efficient single-pass cw second-harmonic generation in periodically poled lithium niobate," Opt. Lett. 22, 1834-1836 (1997). 